\title{
NAO robot fuzzy obstacle avoidance in virtual environment
}

\author{
Octavian Melinte, Luige Vladareanu, Ionel-Alexandru Gal \\ Department of Robotics and Mechatronics, Romanian Academy, Institute of Solid Mechanics
}

\section{Article Info}

Received Jan $2^{\text {nd }}, 2019$

\section{Keyword:}

Fuzzy

NAO robot

Obstacle avoidance

Navigation

\begin{abstract}
The fuzzy inference system for obstacle avoidance developed in this paper is designed for NAO humanoid robot. The fuzzy obstacle avoidance (Fuzzy OA) has been tested in Webots virtual environment and the results showed that this method is almost two times faster than the Naoqi framework obstacle avoidance (Naoqi OA) while the robot is much more stable. Because the fuzzy inference system is a method that relies on trial an error and experience, the obstacle avoidance algorithm is subject to improvements. Future developments will take into account these results and will add other fuzzy inference systems for navigation, in order to get more autonomy for Nao robot.
\end{abstract}

\section{Corresponding Author:}

Luige Vladareanu, Romanian Academy, Institute of Solid Mechanics of the Romanian Academy,

15 Constantin Mille Street, Bucharest, Romania.

Email: luige.vladareanu@ vipro.edu.ro

\section{Introduction}

Humanoid robots are the most interesting and difficult area of robotics. The state of the art research in this area focuses on developing intelligent decision making algorithms, increasing the stability of humanoids robots in unstructured environments, optimizing the path planning, battery efficiency, increase in autonomy etc.

Nao is a humanoid robot which is widely used in the research area due to its good performances, small size, affordable price and the wide range of sensors it is equipped with. Humanoid robots, among which Nao, are known to be difficult to navigate, since motion odometry is computed from the relative motion of legs, which often slip, and thus the odometry error is large and increases rapidly. There are several researches that explored this problem for Nao robots. In [1] a fuzzy intelligent technique for navigation of humanoid robot in obstacle prone zone is presented. The fuzzy inference system used in this case takes four inputs: left obstacle, front obstacle, right obstacle and bearing angle towards the target while, after computation, two outputs are obtained: left and right velocity. In the paper is not mentioned how the front distance is obtained, taking into account that the Nao robot has only two ultrasonic sensors, one in the right side and one in the left side. It is also unclear why left and right velocity are chosen as fuzzy outputs, different velocities of humanoid robot legs does not mean the robot will turn. This assumption can be applied to mobile robots with differential drive, on humanoid robots the only effect is increasing the instability during the walk and maybe making a turn at some point, due to the unstable walk. Another approach for path planning and navigation is in [2]. The fuzzy theory and Markov chains are combined in order to obtain a fuzzy Markov decision process for humanoid path planning. The algorithm takes a input image from Nao camera processes it using Markov chains. The obtained image is sent to a fuzzy system which decides which direction Nao robot should follow. This method works with small objects that can be captured in the camera frame, walking along a wall or getting through a maze with walls bigger that robot can be an impossible task. The image processing is also subjected to a lot of 
noise during the walk, as Nao walk is unstable. A Neural Approach for Robot Navigation based on Cognitive map Learning is presented in [3]. This paper presents a neural network architecture for a robot learning new navigation behaviour by observing a human's movement in a room. There are also a lot of works that study the control of autonomous robots using neural networks, fuzzy, PSO, ANFIS . In [4] a fuzzy logic technique for Romeo Autonomous Vehicle Navigation is proposed, an on- line navigation technique for a wheeled mobile robot in an unknown dynamic environment using fuzzy was developed in [5], a new behaviour-based fuzzy control method for Voyager II mobile robot navigation is presented in paper in [6], a fuzzy discrete event systems framework for the control of sampled data systems that have to fulfil multiple objectives has been introduced by Schmidt K.W. et al. in [7]. Nao and humanoid robot control have also been the object of other research works of the authors, such as Melinte $\mathrm{O}$ et al. in [8] for haptic robots, Wang X et al. for rescue robots [9], Feng Y. in [10], Wang H. for rehabilitation robots in [11] and the results are applied in this paper.

\section{NAO robot control}

The sensor network, Naoqi Framework and CPU architecture make Nao robot an affordable humanoid robot that can be used in different research area. In order to implement the obstacle avoidance control we need to get information from the two front sonars on the robot, process these data and send the crisp outputs to the speed and angle control modules.

In order to navigate in unknown environments the robot has to get information, process them and providing an optimal solution for the task. One particular issue for reaching the target position is avoiding the obstacles encountered in robot's path. There is a lot of research work carried out so far in this field but we will focus into implementing a fuzzy inference system that has a high accuracy and adapts better to our requirements.

The fuzzy algorithm used for obstacle avoidance is a Mamdani inference system. In the fuzzification stage the systems requires to map the inputs from two ultrasonic sensors to values ranging from 0 to 1 using a set of input membership functions. The two ultrasonic sensors are placed on Nao's chest. Each sensor is equipped with transmitter and receiver, with a distance of $7 \mathrm{~cm}$ between the two sonars transmitters. The sonars effective cone is 600 , frequency $=40 \mathrm{kHz}$ and the detection range varies between 0.25 to $2.55 \mathrm{~m}$, with a resolution of $1 \mathrm{~cm}$.

The membership functions represent the Near(N), Medium Near (NM), Medium(M), Medium Far(NF) and $\operatorname{Far}(\mathrm{F})$ linguistic variables of each input membership functions. The fuzzification for each input is the same. The five linguistic functions have a trapezoidal shape. In fig.1 the fuzzification of the three inputs is presented. The range of the sensors is between 0 and $2.5 \mathrm{~m}$, the values over this limit are considered as "Far" and do not influence the output of obstacle avoidance control. We had to take into consideration that the ultrasonic sensors do not read distance information when a obstacle is less than $25 \mathrm{~cm}$ from the robot, the values under this limit are represented as "Near".
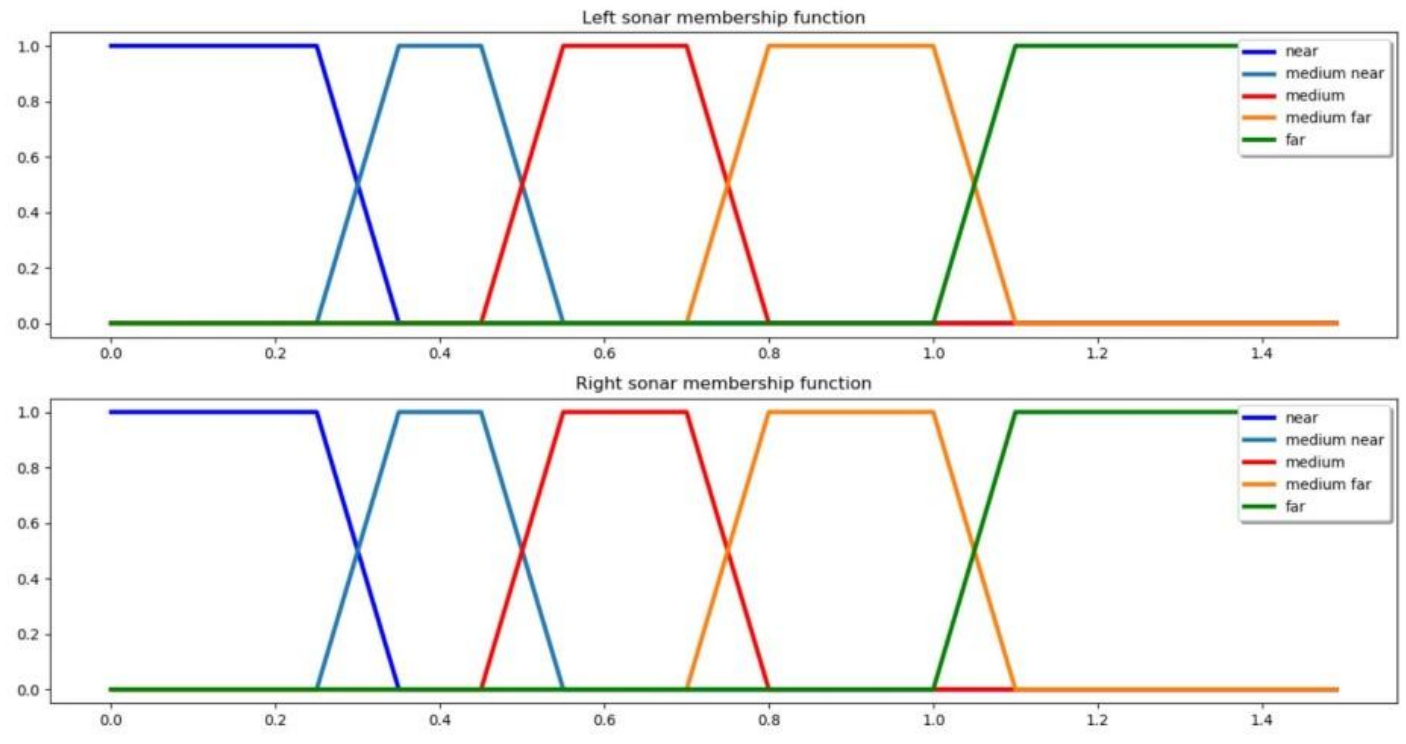

Figure 1. Input membership functions NAO robot(units) 
In figure 3 the output membership functions of the fuzzy set are represented. The outputs of the fuzzy set are the speed and orientation of the robot. Each output membership has five linguistic values. The robot speed subsets are all triangle shape and are defined as: "VSmall"(VS), "Small"(S), "Medium"(M), "High"(H) and "VHigh" $(\mathrm{VH})$. The universe of discourse is between 0 and 1, which represents the size of each step, in robot terms. High values means large steps (increased speed), while small values the opposite, small steps (low speed).

The robot angle subsets are made of four trapezoidal shape subsets and one triangle shape. The trapezoidal subsets are defined as: "Small positive"(SP), "High positive"(HP), "Small negative"(SN), "High negative" $(\mathrm{HN})$, while the triangle subset is defines as "Zero" $(\mathrm{Z})$. The universe of discourse is between -100 and 100 degrees, which represents the turn of the robot when a obstacle is detected.
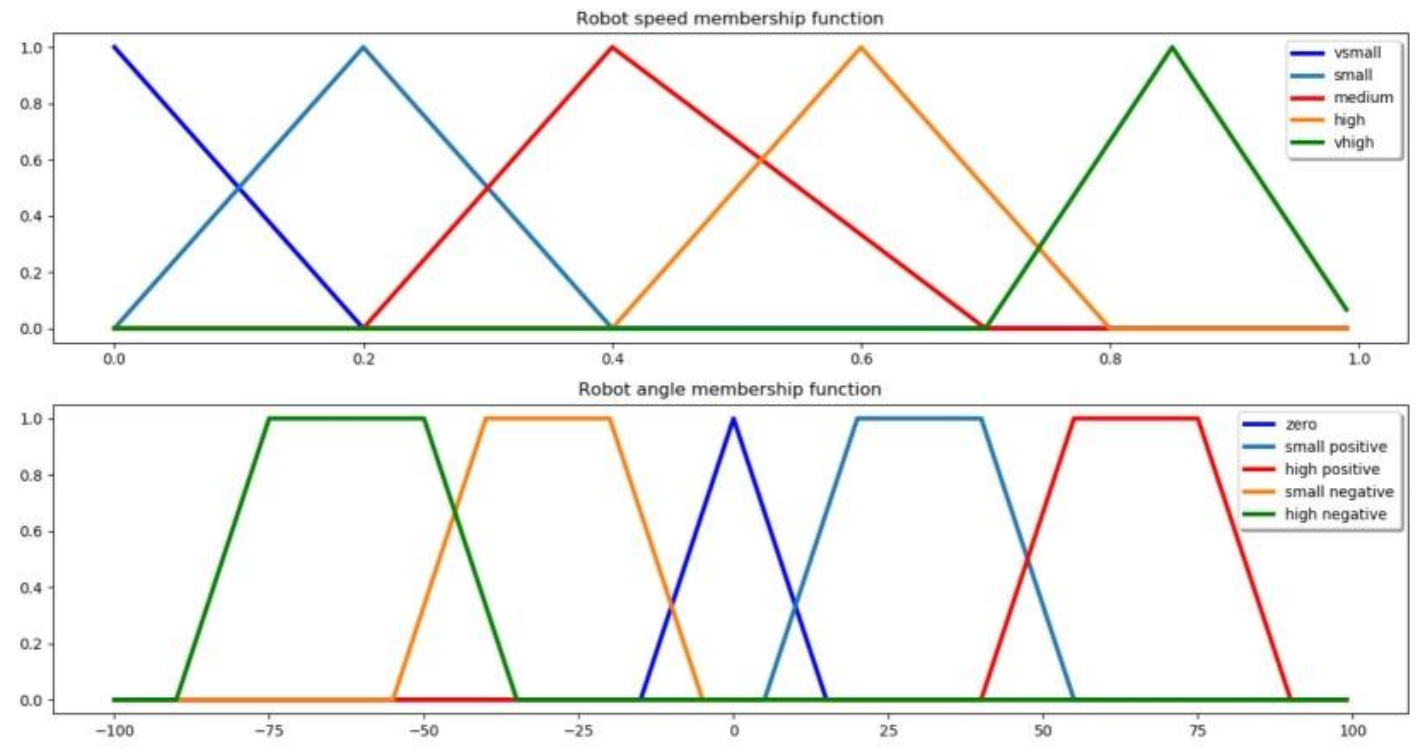

Figure 2. Output membership functions Nao robot(units)

The reasoning above requires a set of rules to be defined. These rules define the relationship between input and output fuzzy membership functions. The general form of these rules are for example: "if input 1 is Near and if input 2 then speed is VSmall and angle is HighNeg". In order to get the fuzzified outputs we developed 25 fuzzy rules based on max aggregation method (“AND").

In table 1 the fuzzy rules are presented. We have two input sensor information as fuzzy membership functions with five linguistic variables each -Near(N), Medium Near (NM), Medium(M), Medium Far(NF) and Far(F)and two fuzzy outputs with five membership functions each- "Very Small"(VS), "Small"(S), "Medium"(M), "High"(H), "Very High"(VH) for speed and "Small positive"(SP), "High positive"(HP), "Small negative"(SN), "High negative"(HN), "Zero"(Z) for orientation.

Table 1. Fuzzy OA rules

\begin{tabular}{|c|c|c|c|c|c|}
\hline LD/RD & N & NM & M & NF & F \\
\hline N & VS-HN & VS-HP & S-HP & S-SP & M-SP \\
\hline NM & VS-HN & S-HN & M-HP & M-SP & H-SP \\
\hline M & S-HN & M-HN & M-SP & H-SP & H-SP \\
\hline NF & S-SN & M-SN & H-SN & H-Z & VH-SP \\
\hline F & M-SN & H-SN & H-SN & VH-SN & VH-Z \\
\hline
\end{tabular}


In fig.3. is presented the activation of each input membership function, for a crisp input of $0.45 \mathrm{~m}$ on the left sensor and $0.7 \mathrm{~m}$. The output membership functions for this input are also presented in fig. 4 together with the deffuzified speed value of $0.46 \mathrm{~m} / \mathrm{s}$ and angle of $47.5^{\circ}$.
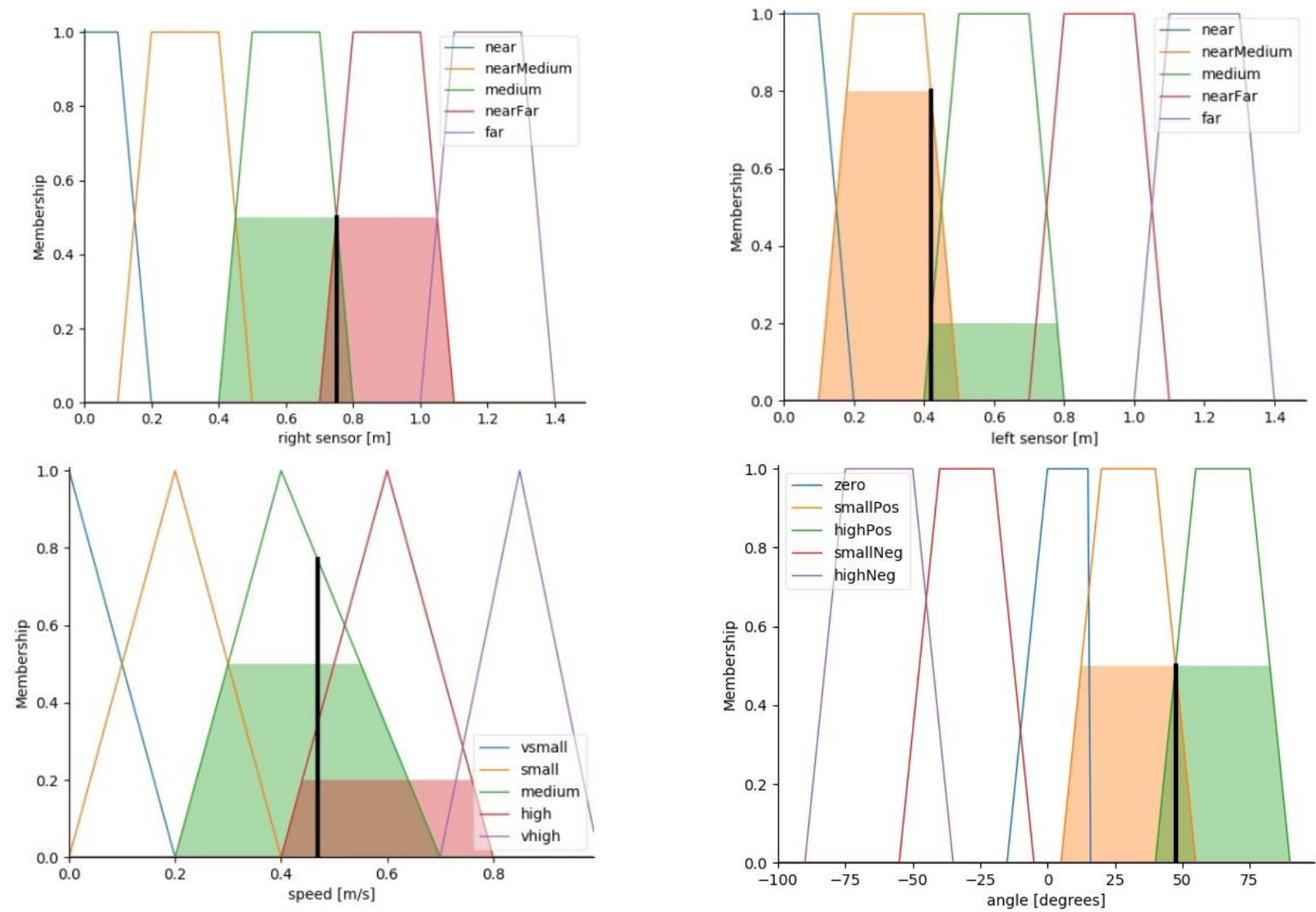

Figure 3. Fuzzy rules set

The defuzzification of each output distribution is obtained using centroid of area method, also called centre of gravity. This method provides a crisp value based on the center of gravity of the fuzzy set. The total area and the centre of gravity of each subset is calculated and then the summation of all these sub-areas is taken to find the defuzzified value for a discrete fuzzy set. In fig. 4, the activation of each fuzzy rule is presented.
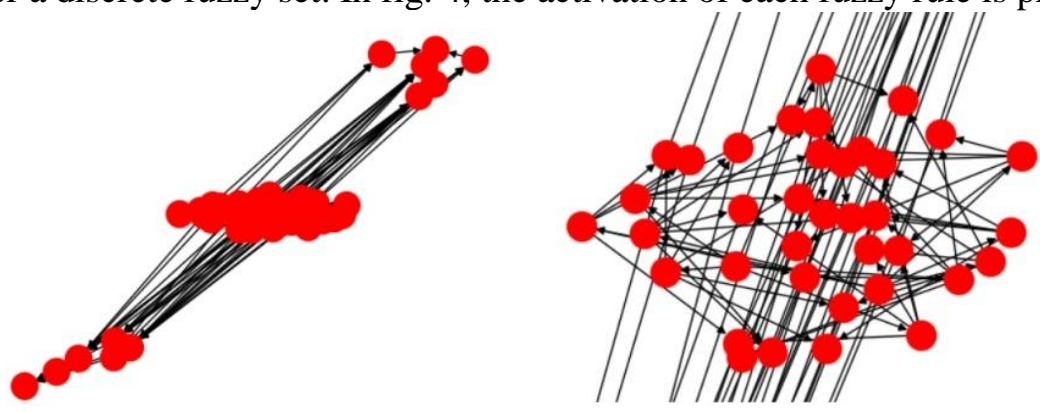

Figure 4. Activation of fuzzy rules

Using the fuzzy inference system described above we have obtained the crisp speed and orientation outputs for our Nao robot. 

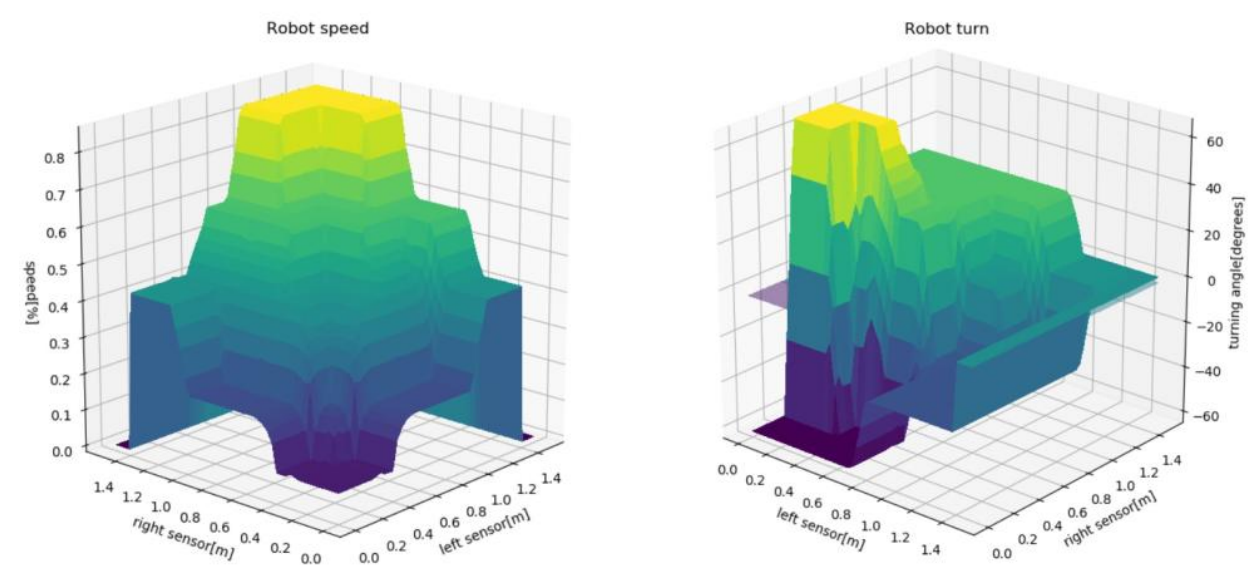

Figure 5. Mesh grid of the relationship between inputs and outputs. ()

In order to map the mesh grid of each output we provided a set of 50 crisp values for each input, evenly spaced over their universe of discourse. The result is a set of 50 crisp values for each output. The relationship between inputs and outputs is plotted in fig. 5 .

\section{Experiment set-up and results}

The experiments took place in Webots, a robot simulator that provides a complete development environment to model, program, simulate and validate robotic researches. Because Nao robot has some stability issues the simulation and validation in a VE is a responsible approach in order to protect the real robot from bumping against surrounding objects or falling during trials. Our experiments consisted in several scenarios, where robot had to walk in a virtual apartment room and avoid objects in his path. Objects in the room had different sizes and shapes. In fig. 6 and fig.7 we present the trajectories of the robot where target position was set in right side and left side of the room. Nao robot is able to avoid all objects in the virtual environment and even able to squeeze between two objects in the room, as we can see in fig.7. We have also tested the robot walking along the wall having to make a decision when came across corners or narrow gaps.

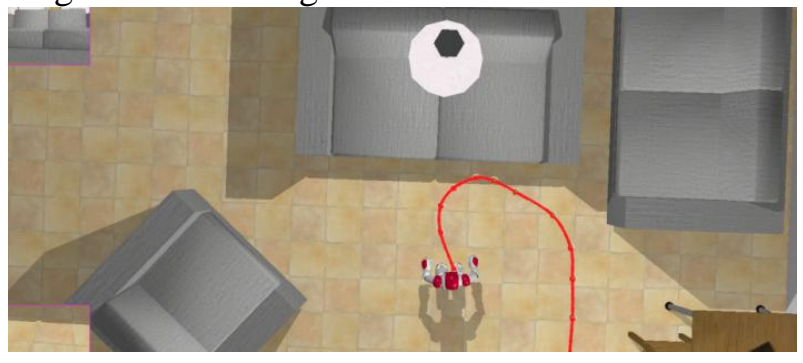

Figure 6. Nao robot walking to the right side of the room

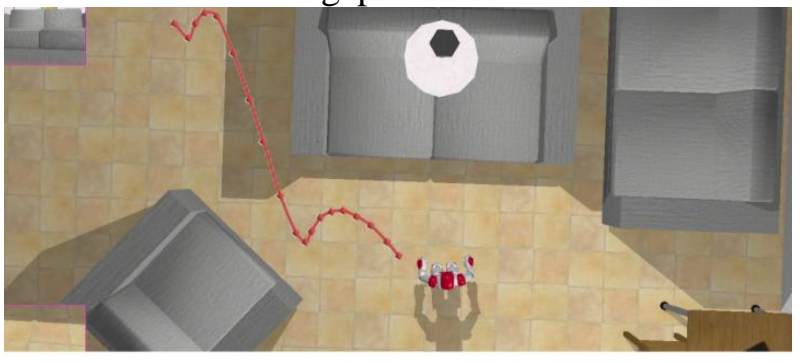

Figure 7. Nao robot walking to the left side of the room

Nao robot has a Naoqi framework that includes a method called Obstacle Avoidance (Naoqi OA), which is part of the ALMotion library. We compared our Fuzzy Obstacle Avoidance (Fuzzy OA) control with this function. In terms of arriving to the same target position our Fuzzy OA was almost two times faster than Naoqi Obstacle Avoidance. The Naoqi OA is a basic obstacle avoidance method which moves the robot backwards and then turning only to the right side when an obstacle is detected. This involves several extra movements, relative to our algorithm, which makes the robot arriving to the target position with a big delay. Due to the Naoqi OA drawback of turning only to the right when an obstacle is detected, we set target positions in the right side of the robot (fig.7) for better comparison. In addition to algorithm low optimization, there are stability issues when using Naoqi OA. We had to run the NAoqi OA simulation several times due to robot falling during the walk. As mentioned before, the robot has some stability issues after 5-10 steps, so if the walk is longer, the stability decreases. In our case, the Fuzzy OA has a reset after 3 seconds of walk, the robot stops for 0.5 seconds, helping to regain its stable posture. 
In the near future, future experiments will take place on the real robot moving in a real environment. Likewise, the fuzzy OA will be subjected to further adjustments and other fuzzy inference systems will be added in order to improve the OA control, goal seeking and stability.

\section{Conclusion and future work}

The fuzzy inference system for obstacle avoidance developed in this paper is designed for Nao robot. The robot has been tested in Webots virtual environment and will be tested on the real robot in the future. The Fuzzy OA is almost two times faster than the NAOqi OA and the robot is much more stable. Because the fuzzy inference system is a method that relies on trial an error and experience, the obstacle avoidance algorithm is subject to improvement. Future developments will take into account these results and will add other fuzzy inference systems, such as goal seeking fuzzy system, in order to get more autonomy for Nao robot. In addition to these methods a decision making algorithm will be needed in order to fire the appropriate method. This algorithm is intended to be developed using deep learning, machine learning or neutrosophic logic.

\section{Acknowledgements}

This work was supported by a grant of the Romanian Ministry of Research and Innovation, CCCDIUEFISCDI, MULTIMOND2 project number PN-III-P1-1.2-PCCDI2017-0637/33PCCDI/01.03.2018, and by KEYTHROB project, number PN-III-P3-3.1-PM-RO-CN-2018-0144 / 2 BM/2018, within PNCDI III, and by the European Commission Marie Skłodowska-Curie SMOOTH project, Smart Robots for Fire-Fighting, H2020-MSCA-RISE-2016-734875. The authors gratefully acknowledge the financial support of Department of Technical Sciences of the Romanian Academy.

\section{References}

[1] Kumar R.A, Parhi D.R., Das H.C., Muni M.K., and Kumar P.B, "Analysis and use of fuzzy intelligent technique for navigation ofhumanoid robot in obstacle prone zone", Defence Technology (2018)

[2] Mahdi F, Kosari A., and Jafarzadeh M., "Humanoid robot path planning with fuzzy Markov decision processes." Journal of applied research and technology 14, no. 5 (2016): 300-310.

[3] Yan W., Weber C., Wermter S., "A neural approach for robot navigation based on cognitive map learning." IEEE International Joint Conference on Neural Networks (IJCNN), pp. 1-8., 2012.

[4] Driankov D. and Saffiotti A.,"Fuzzy logic techniques for autonomous vehicle navigation”. Vol. 61. Physica, 2013.

[5] Faisal M., Hedjar R., Al Sulaiman M. and Al-Mutib K. "Fuzzy logic navigation and pbstacle avoidance by a mobile robot in an unknown dynamic environment." International Journal of Advanced Robotic Systems 10.1 (2013): 37.

[6] Mo H., Tang Q., Meng L., (2013). "Behavior-based fuzzy control for mobile robot navigation", Mathematical problems in engineering, 2013.

[7] Schmidt K.W. and Boutalis Y.S., "Fuzzy discrete event systems for multiobjective control: Framework and application to mobile robot navigation." IEEE Transactions on Fuzzy Systems 20.5 (2012): 910-922.

[8] Melinte O., Vladareanu L.,Munteanu L., Yu H., Cang S., Hou Z.G., Bian G.B., and Wang H.. "Haptic intelligent interfaces for NAO robot hand control.", IEEE International Conference on Advanced Mechatronic Systems (ICAMechS), pp. 50-55, 2015.

[9] Wang X., Wang X., Yu H., Wang H., Lu L., Vladareanu L. and Melinte D.O., "Dynamic analysis for the leg mechanism of a wheel-leg hybrid rescue robot," 2014 UKACC International Conference on Control (CONTROL), Loughborough, 2014, pp. 504-508. doi: 10.1109/CONTROL.2014.6915191.

[10] Feng Yongfei, Wang Hongbo, et al., (2016), Teaching Training Method of a Lower Limb Rehabilitation Robot, International Journal of Advanced Robotic Systems, 2016,13(57): 1-11.

[11] Wang, H., Feng, Y., Yu, H., et al., (2018). "Mechanical design and trajectory planning of a lower limb rehabilitation robot with a variable workspace". International Journal of Advanced Robotic Systems. https://doi.org/10.1177/1729881418776855. 